\title{
Utilizing Computational Machine Learning Tools to Understand Immunogenic Breadth in the Context of a CD8 T-Cell Mediated HIV Response
}

OPEN ACCESS

Edited by:

Lucia Lopalco,

San Raffaele Hospital (IRCCS), Italy

Reviewed by:

Chiara Brombin,

Vita-Salute San Raffaele

University, Italy

Federica Chiappori,

National Research Council

(CNR), Italy

${ }^{*}$ Correspondence: Ed McGowan

ed.mcgowan@isogenica.com Jonathan Hare jhare@iavi.org

Specialty section:

This article was submitted to

Viral Immunology,

a section of the journal

Frontiers in Immunology

Received: 24 September 2020 Accepted: 28 January 2021

Published: 18 February 2021

Citation:

McGowan E, Rosenthal $R$, Fiore-Gartland A, Macharia $G$ Balinda S, Kapaata A, Umviligihozo G, Muok E, Dalel J, Streatfield CL, Coutinho H, Dilernia D, Monaco DC

Morrison D, Yue L, Hunter E, Nielsen M, Gilmour J and Hare $J$ (2021) Utilizing Computationa

Machine Learning Tools to Understand Immunogenic Breadth in the Context of a CD8 T-Cell Mediated HIV Response.

Front. Immunol. 12:609884. doi: 10.3389/fimmu.2021.609884
Ed McGowan ${ }^{1 *}$, Rachel Rosenthal ${ }^{2}$, Andrew Fiore-Gartland ${ }^{3}$, Gladys Macharia ${ }^{1}$, Sheila Balinda ${ }^{4}$, Anne Kapaata ${ }^{4}$, Gisele Umviligihozo ${ }^{5}$, Erick Muok ${ }^{5}$, Jama Dalel ${ }^{1}$, Claire L. Streatfield ${ }^{1}$, Helen Coutinho ${ }^{1}$, Dario Dilernia ${ }^{6}$, Daniela C. Monaco ${ }^{6}$, David Morrison ${ }^{7}$, Ling Yue ${ }^{6}$, Eric Hunter ${ }^{6}$, Morten Nielsen ${ }^{8}$, Jill Gilmour ${ }^{1}$ and Jonathan Hare ${ }^{9 *}$

${ }^{1}$ IAVI Human Immunology Laboratory, Imperial College, London, United Kingdom, ${ }^{2}$ Cancer Evolution and Genome Instability Laboratory, Francis Crick Institute, London, United Kingdom, ${ }^{3}$ Vaccine and Infectious Disease Division, Fred Hutchinson Cancer Research Center, Seattle, WA, United States, ${ }^{4}$ Medical Research Council/Uganda Virus Research Institute (MRC/UVRI) and London School of Health and Tropical Medicine (LSHTM), Uganda Research Unit, Entebbe, Uganda, ${ }^{5}$ Project San Francisco (PSF) Center for Family Health Research (CFHR), Kigali, Rwanda, ${ }^{6}$ Emory Vaccine Center, Emory University, Atlanta, GA, United States, ${ }^{7}$ Bitefirst, South Walsham, United Kingdom, ${ }^{8}$ Department of Health Technology, Technical University of Denmark, Lyngby, Denmark, ${ }^{9}$ IAVI, New York, NY, United States

Predictive models are becoming more and more commonplace as tools for candidate antigen discovery to meet the challenges of enabling epitope mapping of cohorts with diverse HLA properties. Here we build on the concept of using two key parameters, diversity metric of the HLA profile of individuals within a population and consideration of sequence diversity in the context of an individual's CD8 T-cell immune repertoire to assess the HIV proteome for defined regions of immunogenicity. Using this approach, analysis of HLA adaptation and functional immunogenicity data enabled the identification of regions within the proteome that offer significant conservation, HLA recognition within a population, low prevalence of HLA adaptation and demonstrated immunogenicity. We believe this unique and novel approach to vaccine design as a supplement to vitro functional assays, offers a bespoke pipeline for expedited and rational CD8 T-cell vaccine design for HIV and potentially other pathogens with the potential for both global and local coverage.

Keywords: CD8 T-cells, HIV, T-cell epitopes, vaccines, machine learning

\section{INTRODUCTION}

Since the Human Immunodeficiency Virus (HIV) was first identified, 77.3 million people have become infected of which 35.4 million people subsequently died (1). Decades of research has enabled a comprehensive understanding of the structure, genetics, mechanism of infection, immune control and immune escape to emerge, resulting in novel targets for interventions, both as therapeutic targets, and for prophylaxis in the form of a broadly efficacious vaccine (2).

The structure of HIV lends itself to the development of vaccines that target the dominant surface glycoprotein gp120 and lead to the development of broadly neutralizing antibodies (3). 
Approaches to develop immunization regimes that will bias the development of this class of antibodies to provide prophylactic protection against HIV infection are under development with the first products entering clinical assessment (4). However, natural control of HIV viral load following the acute viral load burst is associated with a T-cell mediated response (5) and this suggests that a vaccine designed to raise $\mathrm{T}$-cell responses may have efficacy if it is targeted to defined antigenic regions (6) including those with integral networked topology (7).

There are currently a number of T-cell vaccine candidates that utilize a variety of novel design approaches being tested in human clinical trials. The HIV Conserved vaccine (HIVCON) utilizes a conserved mosaic approach whereby regions of the proteome that have been identified as conserved within available databases are arranged in a specific regimen to both elicit T-cell responses to potential epitopes present within these regions, whilst limiting immunogenicity to the necessary joining or junctional regions (8). A second approach is to assemble known T-cell epitopes in a mosaic approach, whereby composite proteins are created to include common T-cells epitopes in a polyvalent design (9). A third approach, HIVACAT Tcell Immunogen, involves the construction a chimeric protein encoding 16 continuous segments of HIV derived from Gag, Pol, Vif, and Nef (10). There are pros and cons to all these approaches, but a potential caveat to utilizing conserved regions of the proteome is that historically pathogen diversity has been measured as the similarity or dissimilarity of sequences to each other, however a vaccine design should factor in how this pathogen sequence conservation is viewed by the host immune system.

Development and implementation of predictive models is becoming more commonplace as tools for candidate antigen discovery (11). This is highly relevant for HIV vaccine discovery where there is a staggering amount of complexity posed by diversity observed within individuals (12), within and between clades $(13,14)$ and within populations (15) making it a formidable challenge for rational T-cell vaccine design.

Here we present an in silico approach that complements the vaccine design strategies through the identification of HLA restricted antigenic regions within diverse HIV sequences based upon modeling of HLA restricted responses within individuals and linking these to disease progression via samples obtained from IAVI Protocol C, a longitudinal acute HIV infection study in east and sub-Saharan Africa covering multiple incident infection subtypes (16). We show that within a population, although HLA sequences show high levels of polymorphism, there are conserved, and over represented alleles associated with the $>80 \%$ of the population covered within the study. In this study, we propose the use of the artificial neural network, NetMHCpan $(17,18)$ as a proxy to identify putative CD8 Tcell epitopes contained within the HIV transmitted founder virus (TFV) identified from the Protocol C clinical cohort of sub Saharan and East Africa. Using the transmitted founder virus sequence for relevant vaccine design is a well-established concept (19) and exploiting these predicted peptide/HLA interactions to generate additional novel metrics of HIV diversity adds another layer of information to facilitate vaccine design.
TABLE 1 | Distribution of input transmitted founder proteome data.

\begin{tabular}{lll}
\hline Clade & $\boldsymbol{N}$ & Distribution \\
\hline A & 44 & Kenya (19), Rwanda (18), Uganda (6), Zambia (1) \\
C & 38 & Kenya (2), Rwanda (1), Uganda (2). Zambia (33) \\
D & 27 & Kenya (3), Uganda (24) \\
Recombinant & 16 & Kenya (6), Rwanda (4), Uganda (8)
\end{tabular}

Number of sequences from each country listed in parentheses.

We believe that the size of the study cohort used in this investigation enables an extrapolation and scaling of the approach to global populations to enable a rationalized isolation and prediction of antigenic epitopes for any disease where a T-cell response is dominant in its control. By further informing vaccine strategies to focus the immune system against particular pathogens, incorporating potential immune recognition information into established models may increase the likelihood of success (20).

\section{MATERIALS AND METHODS \\ Cohort Characteristics}

HLA profiles were evaluated from HIV+ volunteers enrolled in two IAVI-sponsored clinical cohorts. IAVI Protocol $\mathrm{C}$ is a prospective vaccine preparedness cohort studies of HIV-1 antibody negative heterosexuals or men who have sex with men in a Uganda Virus Research Institute/Medical Research Council/Wellcome Trust HIV-1 acquisition cohort study, and in a heterosexual sero-discordant couple's cohort study in Rwanda. Subjects were given HIV counseling, condom provision and regular HIV testing either monthly or quarterly. Those who seroconverted to HIV-1 were screened for stage of primary HIV1 infection (16). IAVI Protocol G was a cross-sectional cohort of $\sim 2,000 \mathrm{HIV}$ positive individuals enrolled at 13 sites around the world in order to identify circulating broadly neutralizing antibodies (21).

\section{Near Full Length Transmitted Founder Genomes}

The selection criteria for inclusion in the generation of near full length transmitted genomes is as previously described (22). For this analysis, 125 Near Full length transmitted Founder genomes were evaluated from across Africa (Table 1).

\section{HLA Distribution}

The HLA binding predictor NetMHCpan was used to identify putative epitopes in 125 Transmitted Founder HIV-1 gag sequences derived from a cohort in Zambia (23). The distance between two sequences was defined as the percent of mismatched amino-acids in each 9 mer, summed across all 9 mers spanning the entire protein (i.e., a 500 aa protein contains $492 \times 9$ mers, each overlapping by $8 \mathrm{aa}$ ). This distance is dependent on sequences being aligned and therefore sequences sometimes contain gaps indicating insertions; this treats each gap character as an aa. Using this metric, the distance for the entire protein 
or for a subset of the 9 mers was determined; the epitopebased distance included only 9 mers in the alignment that were predicted to bind to at least one HLA allele. Binding was based on a threshold of $500 \mathrm{nM}$, though sensitivity analyses showed similar results with different thresholds.

\section{Model Implementation}

For each virus proteome a NetMHCpan simulation is performed for each of 46 Human Leukocyte Antigen (HLA) sequences. The 46 NetMHCpan result files for a virus proteome are then filtered to extract the peptide, HLA and rank binding where the rank binding is $\leq 2$ [lower value is stronger binding $(24,25)$ ]. This data is then loaded into a PostgreSQL database where an analysis tool is implemented in SQL stored procedures to identifies key peptides which appear in at least $\mathrm{X}$ viruses strains. The conservation metric $\mathrm{X}$ is defaulted to $2.2 \%$ of the total number of viruses initially being analyzed. The analysis tool then selects the virus that contributes the most of these key peptides. The selected virus and associated key peptides are then removed from the process and the next virus that contributes the most of the remaining key peptides is selected. The ranking process continues until all the key peptides are accounted for. The ranking results are then available to view or download at https://ibpt.iavi.org.

For comparison, set-building was performed a second time using randomly selected strains instead of choosing the strain that resulted in the greatest increase of peptide coverage.

\section{HLA Adaptation Analysis}

HLA adaptation analysis was performed as previously described (26). Briefly, each of the 319 peptides in the peptide set was aligned to the Zambian consensus sequence corresponding to the protein they were derived from and to HXB2. HLA adaptation was assessed using a list of statistically significant viral amino acid-HLA allele associations for Gag, Pol and Nef, previously described in Carlson et al. (27), as well as a new list generated for Rev, Tat, Vif and Vpr based on 295 sequences derived from chronically-infected individuals from Zambia plus 237 subtype $C$ sequences downloaded from LANL (unpublished). A peptide was identified as adapted when the residue was positively correlated with the HLA or was any other residue other than the one negatively correlated with that HLA or the consensus (referred to as non-adapted). The correlation of residues to HLA was determined based on the number of HLA-linked polymorphisms relevant to the HLA alleles repertoire, as well as the number of polymorphisms located within well-defined CTL epitopes restricted by HLA alleles.

\section{IFN- $\gamma$ ELISPOT}

The predicted peptides were evaluated for ability to induce T-cell responses by IFN- $\gamma$ ELISPOT using bi-specific expanded CD8 Tcells as previously described (28). Briefly, PBMC were thawed and cultured in RPMI/10\%FBS media supplemented with IL-2 (Sigma $50 \mathrm{U} / \mathrm{mL}$ final concentration) and a CD3/CD4 bispecific antibody (Genscript) to expand CD8 T-cells. On Day 7 of expansion the CD8 population was assessed by Human IFN- $\gamma 96$ well ELISPOT (Mabtech) as per manufacturer's instructions. Peptide pools for 319 peptides were prepared as an $11 \times 11 \times 113 \mathrm{D}$ matrix with
TABLE 2 | Volunteers selected for determining HLA coverage within a population.

\begin{tabular}{|c|c|c|c|c|c|c|}
\hline ID & LA-A & LA-A & LA-B & HLA-B & HLA-C & HLA-C \\
\hline 58 & $x^{2}-5$ & & 5 & & & (2) \\
\hline S & & & & & & \\
\hline 0С305154 & 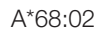 & 等 & 3 & & & 1 \\
\hline S & 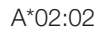 & 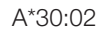 & 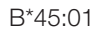 & & & \\
\hline 0С305125 & $A^{\star} 23: 01$ & $A^{\star} 34: 02$ & $\mathrm{~B}^{\star} 08: 01$ & $B^{\star} 15$ & & $\mathrm{C}^{\star} 08: 02$ \\
\hline 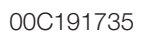 & $A^{\star} 33:$ & A $T$ & $D$ & $\mathrm{~B}^{\star}$ & & \\
\hline & $A^{\star} 23$ & $A^{*}$ & 年 & & & \\
\hline של & $A^{*} 01: 01$ & 34 & $B^{*} 15: 03$ & 1 & 2 & 01 \\
\hline 5 & $A^{\star} 29: 02$ & $A^{\star} 30: 02$ & $\mathrm{~B}^{\star} 4$ & $R^{\star}$ & $\mathrm{C}^{\star}$ & $\mathrm{C}^{\star}$ \\
\hline OC365007 & $A^{\star} 26: 01$ & $A^{\star} 29: 02$ & $B^{\star} 13: 02$ & $\mathrm{~B}^{\star} 8$ & $\mathrm{C}^{\star} \mathrm{C}$ & $\mathrm{C}^{\star} 06: 02$ \\
\hline & $\pi 0$ & & 1 & & & \\
\hline D0G27009 & $A^{*} 02: 05$ & 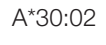 & 2 & $\mathrm{~B}^{\star}$ & $\mathrm{C}^{\prime}$ & $C^{\star} 08: 02$ \\
\hline OG27188 & $A^{*} 02: 05$ & $A^{\star} 30: 01$ & $3^{\star} 07: 02$ & $B^{\star} 27: 03$ & $\mathrm{C}^{\star} 02: 02$ & $\mathrm{C}^{\star} 07:$ \\
\hline
\end{tabular}

each peptide occurring in 3 unique pools. Positive responses were defined as the mean replicate count minus the mean background (mock) count where the mock controls must be $<50 \mathrm{SFU} / 10^{6}$ PBMC and the media only wells $<5$ SFC/well).

\section{Statistical Analysis}

Statistical analyses were carried out using Prism version 6 (GraphPad Software, Inc., La Jolla, CA, USA). Python, Numpy and matplotlib were used to perform the Principal Component Analysis (PCoA).

The differences in sequence coverage determined by the different model parameters were assessed using Area Under Curve analysis and differences in the predicted coverage of each sequence was evaluated using a Kolmogorov-Smirnov test. For experimental ELISPOT data, normal distribution of data was assessed by the Shapiro-Wilk test. ELISPOT responses were compared by Mann-Whitney Test. Spearman correlation was used to assess relationships between ELISPOT responses and sequence priorities and coverage.

The data can be accessed through dataspace.iavi.org.

\section{RESULTS}

\section{HLA Distribution Within Specific Populations}

HLA distribution provides an important metric describing population diversity and correlates with the breadth of viable immune recognition within that population, which is relevant to both immune protection against pathogens and vaccine design strategies. Within Protocol C, all participants were screened for HLA composition upon enrollment and Figure 1 reflects the diversity of HLA Class I alleles within Protocol C (16) at a 2 field (4 digit) level of characterization (29).This data represents the HLA diversity of 613 participants and the prevalence of the HLA A, B, and C alleles is displayed as the relative percentage of the cohort. 


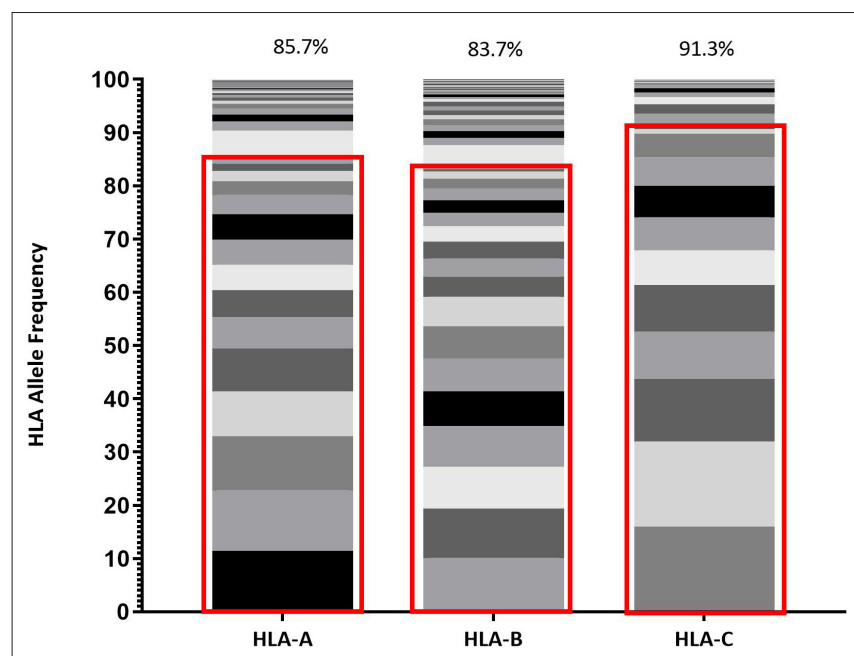

FIGURE 1 | Frequency of each HLA Class I allele (HLA-A, HLA-B, and HLA-C) represented within IAVI Protocol C Alleles. Red boxes demarcate the allele frequencies contained within 13 pre-selected volunteers (Table 2) with percentage coverage listed above each stacked histogram plot. Seventeen Individual alleles contribute to HLA-A analysis, 21 Individual alleles contribute to HLA-B analysis, and 13 Individual alleles contribute to HLA-C analysis.

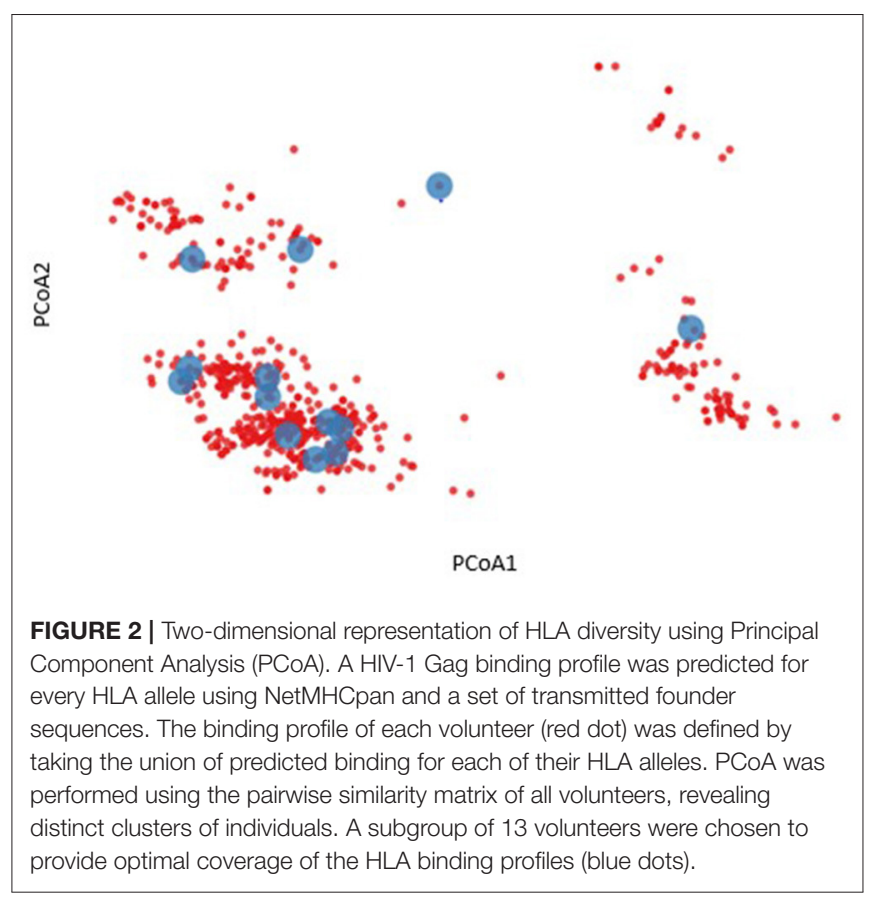

As cumulative sets of HLA alleles are removed (starting with the least frequent alleles) there is minimal loss of epitope binding coverage observed $(<10 \%)$ until a key inflection point is reached, leading to a precipitous loss of coverage, concordant with the frequency of the HLA alleles that are removed. Interestingly, the trend of minimal coverage loss at a minimal HLA frequency is observed independent of the size of the predicted peptide set with a comparable pattern observed for libraries of 300, 250, 200 and 150 peptides suggesting that while the HLA allele binding profile is peptide specific, it may also be independent of the peptides if a sufficient number are used.

\section{Development of a Predictive Model for HIV Diversity}

Using NetMHCpan (at a 1\% Binding Threshold), predicted 8, 9, and 10 mer epitopes were derived from TFV gag sequences $(N=125)$ obtained from HIV-infected volunteers enrolled in IAVI Protocol C, and identified in association with the HLA alleles present (listed in Table 1). Initial model development utilized a 1-select parameter where peptides were considered individually to determine the best coverage. This resulted in the prediction of 6,562 peptides (Supplementary Table 2) and no difference in best coverage mapping vs. random selection by Kolmogorov-Smirnov test $(p=0.4670)$ was observed. Subsequent analysis of this model revealed that 4,812 (73\%) of these peptides were either unique to an individual gag sequence or present in only two gag sequences. If only peptides that were present in $\geq 3$ virus sequences (3-select best) were considered, this led to the prediction of 1,750 peptides $(26.7 \%$ of the 1 select best model), which was shown to be more effective at mapping coverage than randomly selecting peptides $(p<0.0001)$ (Supplementary Figure 2, Supplementary Table 2). 


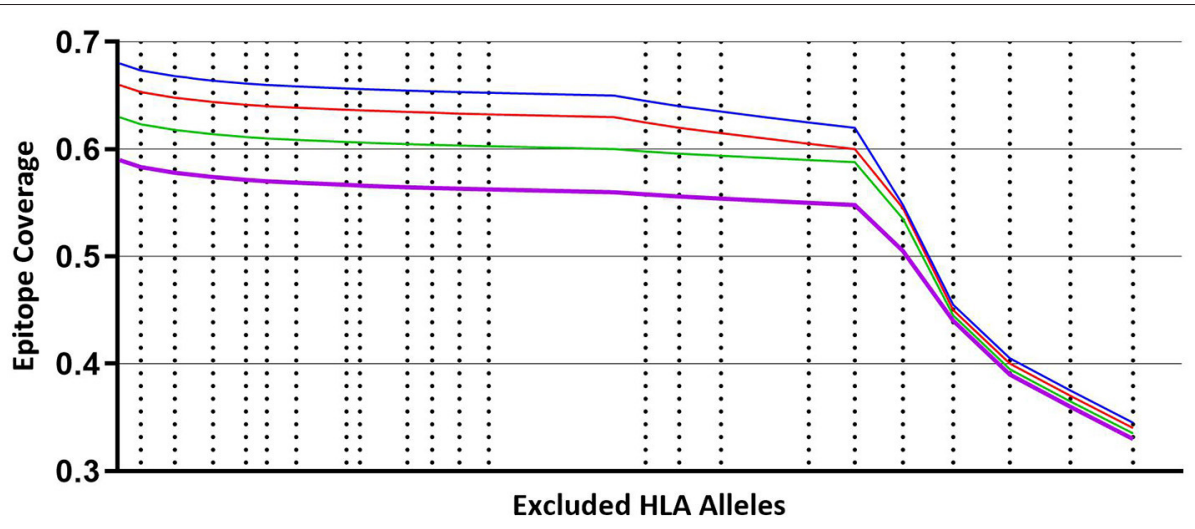

FIGURE 3 | Coverage per predicted peptide calculated against a defined set of HLA alleles. Size of segments on X axis from left to right represents cumulative, combined HLA allele frequencies that are iteratively removed from the analysis, starting with least frequent alleles. Blue line-modeling using predicted 300 peptides. Red line-modeling using predicted 250 peptides. Green line-modeling using predicted 200 peptides. Purple line-modeling using predicted 150 peptides.

Further model development evaluated the effect of varying the binding threshold on the predicted outcomes. The binding threshold is a measurement of confidence that a predicted peptide will associate with the prescribed HLA, for example a $1 \%$ binding threshold factors in a $1 \%$ false positive rate. Running the model whilst varying binding thresholds at $0.5,1$, and $2 \%$ resulted in the identification of $955,1,750$ and 3,023 peptides, respectively (Supplementary Table 2). No difference was observed in coverage when the $1 \%$ binding threshold was set to a less stringent $2 \%$ or a more stringent $0.5 \%$ ( $p=1$ and $p=0.6430$ ), therefore a $1 \%$ binding threshold was selected for all future analyses to maximize coverage whilst being able to distinguish additional conserved epitopes (Supplementary Figure 2).

\section{Modeling of HIV Diversity for Full Length Transmitted Founder Proteomes}

These same parameters (1\% Binding Threshold, Rank Binding $\leq 2$, Peptide Conservation $\geq 2.2 \%$ ) were then applied to analyze 125 Transmitted Founder proteome sequences (excluding envelope) derived from IAVI's Protocol C (see Tables 1, 3 for input sample data and model parameters). The initial evaluation identified 14,953 predicted peptides occurring with a frequency of $2.2 \%$ in our population. This peptide set covers all predicted affinities and coverages and may represent multiple HLA interactions/peptide. To evaluate the distribution of affinities to the primary associated HLAs with Rank Binding scores were assessed (Figure 4A). Rank binding is an alternative metric for HLA:peptide affinity that can be deployed in order to normalize the large diversity in the range of predicted binding values for the different HLA molecules and therefore limit bias derived from over-represented HLA (18). Rank binding assigns each peptide a score with peptides annotated as a strong binder if their score is $<0.5$ or a weak binder if the score is $0.5-2.0$.

To further control for potential bias within the peptide-HLA interactions, the peptides were then analyzed by both affinity and Rank Binding to all predicted HLA interactions (Figure 4B) and
TABLE 3 | Model parameters.

\begin{tabular}{ll}
\hline Parameter & Values \\
\hline Binding threshold & $1 \%$ \\
HLA allele contributions & All HLA alleles from 13 individuals (Table 1) \\
HLA haplotype weighting & 0 \\
Rank binding & $<2.0$ \\
Peptide conservation (\%) & 2.2 \\
Peptide length & $8,9,10$, and 11 mers
\end{tabular}

the frequency that these peptides occurred in the population in the context of the specific HLA alleles (Figure 4C).

This analysis identified a range of predicted binding profiles for the different peptide-HLA interactions (see Supplementary Table 1 for full HLA allele identities). HLA$A^{*} 02: 02, H_{L A}-A^{*} 31: 04$, and HLA-B*15:03 were identified as having particularly high predicted affinity peptide interactions, whereas HLA-B*14:03, HLA-B*15:10, and HLA-C*04:01 have much lower predicted affinity peptide interactions. This differential pattern of binding may be explained due to the large diversity in the range of predicted binding values for the different HLA molecules. When plotted using the Rank Binding metric these differences are less pronounced although trends of stronger associations to specific HLA alleles remain.

Implementing these frequency and binding thresholds to identify HIV-specific predicted CD8 T-cell epitope peptides can be used as a functional metric to assess HIV diversity. By assuming that these predicted peptides provide a novel tool for ranking HIV proteome diversity, it is possible to assign a coverage gain value to each sequence and then utilize those values to rank each sequence for the coverage it provides within the sample population. By implementing these calculations, it is then possible to identify the sequences that are necessary to obtain the optimum level of epitope restricted sequence coverage. 

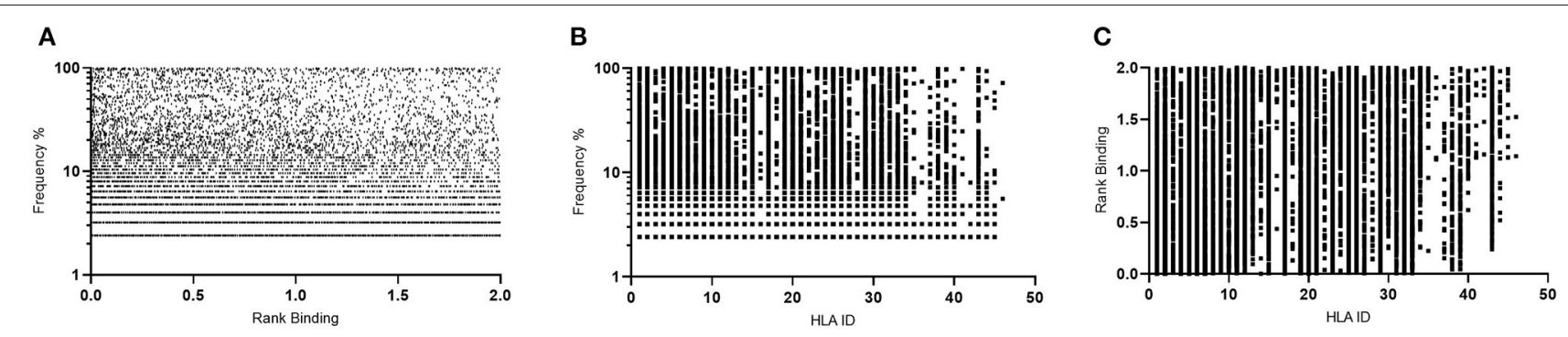

FIGURE 4 | Affinity plots for all predicted peptides with conservation of $\geq 2.2 \%(n=14,953)$. (A) - Predicted peptide affinity (Rank Binding) vs. primary associated HLA. (B) - Predicted peptide affinity (Rank Binding) vs. peptide frequency within transmitted founder proteome. (C) - Predicted peptide frequency vs. primary associated HLA.

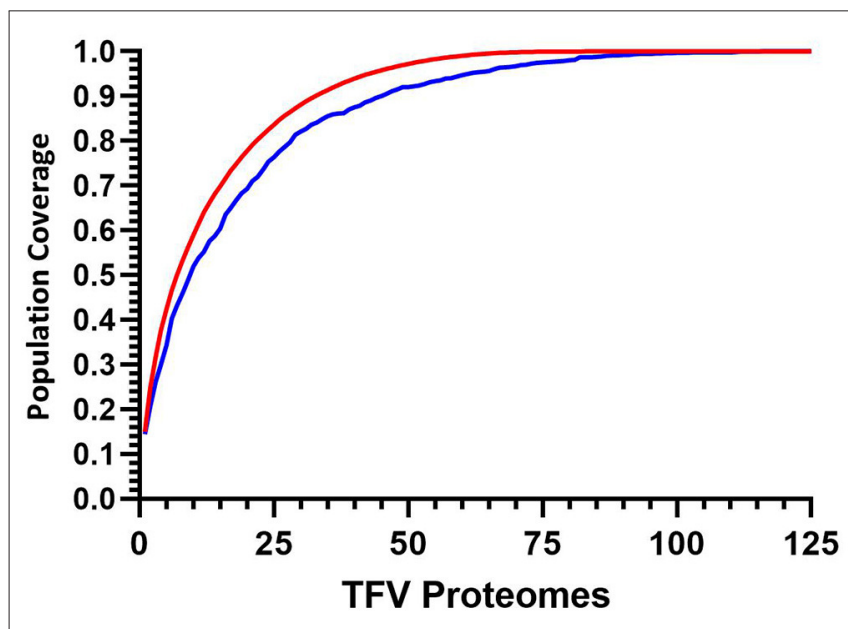

FIGURE 5 | Cumulative coverage distribution plots of full length transmitted founder gag sequences using a 3-select coverage model and a 1\% Binding Threshold, 3-Select best (red), and 3-Select random (blue). P-values calculated using Kolmogorov-Smirnov test.

The implementation of this model can then be used to target and prioritize individual proteomes. Figure 5 illustrates how for 125 transmitted founder virus proteomes, achieving $90 \%$ coverage requires 33 prioritized viruses, which decreases to 22 and 16 viruses if 80 or $70 \%$ coverage is desired, respectively (data not shown). Importantly, $\sim 40 \%$ more viruses are required to achieve $90 \%$ coverage if sequences are randomly selected $(n=45$ $p<0.0001)$.

\section{In silico Characterization of Predicted Peptides}

Whilst evaluating peptides at a prevalence of $\geq 2.2 \%$ is desirable from the perspective of understanding population coverage, it is more challenging to map potential regions of the proteome for anti-HIV T-cell specificities due to the large levels of redundancy and overlap in evaluating each HLA/epitope interaction. By selecting HIV sequence coverage as the primary parameter and predicted affinity as a secondary characteristic the peptide library should contain both predicted high and lower affinity epitopes with optimum coverage, that may have functionality if represented at high enough abundance. Through further stratifications of the predicted peptide set to limit sequence overlap, and through assigning a minimum population coverage of $40 \%$ (selected to maintain sequence conservation and not introduce multiple sequence variations) resulted in the identification of 957 peptides. Of these peptides, an unbiased subset of 319 peptides were selected at random from across the proteome for further in silico and in vitro characterization.

HLA adaptation in a particular epitope is defined as the presence of a particular residue that has been statistically linked to an individual HLA, indicating a process of immune selection in that context (26). Vaccine design utilizing conserved epitopes may unwittingly overlook the observation that not all epitopes in the transmitted virus will be consensus and in fact, some may actively promote CTL escape (30). The peptides identified by the 3-select model were evaluated for predicted HLA adaptation as previously described (26). Of these peptides 75/319 were identified as containing a residue that was adapted, although interestingly the predicted adaptation was against alternative HLA alleles not predicted by the model for $70 / 75$ predicted peptides with only 2 out of 5 adapted peptides associating to the primary HLA allele (Supplementary Table 4).

\section{Predicted Peptide in vitro Characterization}

To confirm that the selected subset of predicted peptides were recognized by anti-HIV specific T-cells, IFN $\gamma$ ELISPOT assays were performed using a 3D Matrix approach described elsewhere (31). The peptides were evaluated in samples from $23 \mathrm{HIV}+$ volunteers at a single time point $\sim 12$ months post-estimated date of infection to determine the contribution of individual HLA and input sequences and correlate these metrics to observed Tcell responses. These volunteers were identified for whether their transmitted founder sequences were included (Group "Seq In"-10 volunteers) or excluded in the modeling analysis (Group "Seq Out" 13 volunteers). ELISPOT responses were also evaluated at a second time point $\sim 60$ months post EDI, although this data was not included in the analysis, Supplementary Table 5).

To evaluate whether the model introduced bias from volunteers who contributed their transmitted founder sequence compared to volunteers whose sequence was not included, 

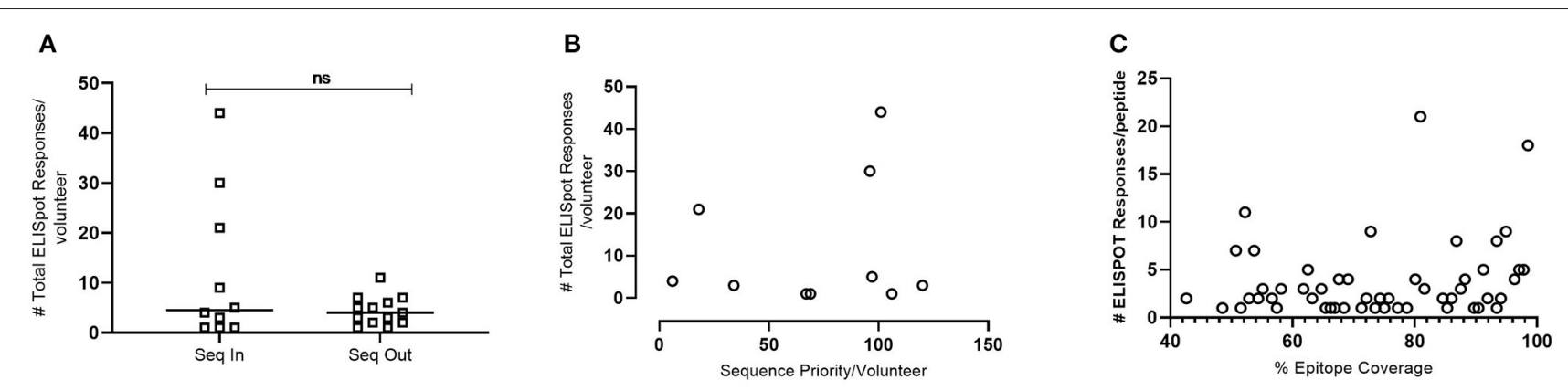

FIGURE 6 | IFN $\gamma$ ELISpot responses observed in HIV+ Volunteers. (A) - Number of total ELISpot responses observed in volunteers whose transmitted founder proteome sequence was included within the in-silico prediction (Seq In: $N=10$ ) and volunteers whose transmitted founder proteome sequence was not included within the in-silico prediction (Seq Out: $N=13$ ). Shapiro-Wilk values Seq In: $W=0.7887, p=0.0008$. Seq Out: $W=0.8976, p=0.0315$. Mann-Whitney test, $p=$ 0.6215. (B) - Correlation of total number of ELISpot responses in volunteers whose transmitted founder proteome sequence was included within the in-silico prediction against the order of priority the sequence was predicted to occur (Spearman Correlation; $r=0.1356, p=0.2209$ ). (C) - Correlation of total number of ELISpot responses in volunteers whose transmitted founder proteome sequence was included within in silico prediction against the \% coverage each epitope represented (Spearman Correlation; $r=0.2695, p=0.0357$ ).

IFN $\gamma$ ELISpot responses were analyzed at 12 months postestimated date of infection. The results indicated no significant difference in the median number of responses per volunteer (median responses/volunteer $\mathrm{n}=4.5$ group Seq In vs. median responses/volunteer $n=4$ group Seq Out, Figure 6A). Further analysis revealed that there was no bias in responses toward the volunteers with sequences predicted to contribute the most coverage vs. those volunteers whose sequences contributes less to coverage (Figure 6B). Assessing the number of individual ELISPOT responses per peptide revealed a trend toward increasing number of responses as the conservation of the peptides increases, although this correlation was not significant (Figure 6C).

\section{DISCUSSION}

We propose that through the addition to the predictive algorithm NetMHCpan, two novel parameters are defined that can be exploited to aid the rational selection of $\mathrm{T}$ cell vaccine immunogens. The first parameter confers the ability to assign a diversity metric to the HLA profile of individuals within a population. The existing metrics of 2-field characterization of HLA alleles enables frequencies of alleles to be calculated but has several limitations when considering HLA diversity/similarity. A clear limitation is that the peptide binding profile of two alleles may not be strongly associated with the similarity of their 2-field allele representation (32). A second method for characterizing HLA allele diversity involves the assessment of the amino acid sequence of the MHC protein with a focus on the peptide binding groove (33). Building on this idea, an alternative, advantageous approach to assessment of the diversity of the HLA frequency may therefore be to use computationally predicted peptide binding of the HLA alleles based on machine learning algorithms trained on functional binding data as well as the amino acid sequences of the HLA proteins (17). We propose an alternative metric of HLA diversity that utilizes the predicted binding affinity of a reference amino acid sequence to assign each HLA allele an individual binding score. By evaluating the individual HLA profiles of individuals in a studied cohort, it is then possible to calculate a combined HLA diversity metric. Using these values, individual volunteers can be mapped within specific populations and distance scores calculated between each allele and each volunteer. Using this approach, we have demonstrated that it is possible to select individuals within a cohort that are "representative" of the population from which they are drawn. Implementing this stratification of volunteers may have implications for the design of smaller experimental clinical trials.

The second parameter is a metric for HIV diversity determined through the perspective of predicted binding of putative CD8 T-cell/HLA epitopes. Previous evaluations of $\mathrm{HIV}$ diversity rely on sequence clustering and alignments to order individual sequences. This alignment is appropriate for comparing the actual sequence of a virus genome or proteome, however this approach is limited for evaluating how an individual may recognize a specific proteome. By considering sequence diversity in the context of an individual's HLA profile and therefore potential CD8 T-cell immune repertoire, an additional diversity metric can be layered to represent how an individual may be predicted to view a virus proteome and through combining the in-silico metrics, it is possible to rank HIV proteome sequences by the coverage they provide within the population across individuals. This ability to rank sequences according to putative immunogenic breadth additionally enables the interpretation of functional immunological killing assays like the viral inhibition assay $(34,35)$. Traditionally these assays have been interpreted as a binary assessment of the number of viruses inhibited. Using these novel metrics, it would now be possible to assign a population coverage score to each virus or panel of viruses and as such be able to provide an estimate as to the potential anti-virus killing activity of a volunteer based on the pattern of viruses they can inhibit. 
IFN $\gamma$ ELISpot analysis using the peptides predicted by the model revealed that there was no significant increase in the number of ELISpot responses/volunteer if the individual's TFV proteome sequence was included in the prediction compared to the number of responses/volunteer if an individual's TFV proteome was not included. This data indicates that using a subset of samples for prediction has not created any inward bias toward the input source but is representative of the population. The frequency of responses observed in this study for both groups are lower than those previously reported (36-38), however this reflects the increased stringency incorporated into the development of this peptide set whereby only peptides with a predicted coverage $>40 \%$ were included. By way of comparison, the conservation threshold for the peptides evaluated by Kunwar et al. (36) and Sunshine et al. (38) were 15 and 5\%, respectively, with a response rate/volunteer of 7 and 12 epitopes, respectively.

This hypothesis indicates that through understanding the conservation, adaptation and functional score assigned to any population of target sequences, it is possible to embed this metric within algorithms to fully evaluate potential immunogenicity within the context of sequence conservation and HLA allele frequency and may contribute to expedited vaccine design and iterative testing strategies aimed at inducing protective CD8 mediated T-cell immunity. The principals underpinning this approach have applicability to other disease models and geographies for which comparative input data is available and protective CD8 responses are desirable.

\section{DATA AVAILABILITY STATEMENT}

The datasets presented in this study can be found in online repositories. The names of the repository/repositories and accession number(s) can be found in the article/Supplementary Material.

\section{ETHICS STATEMENT}

The studies involving human participants were reviewed and approved by the local ethics review boards, including the Kenya Medical Research Institute Ethical Review Committee, the

\section{REFERENCES}

1. Sheet F, Day WA, People V. UNIAIDS Website. (2018) 1-6. Available online at: http://www.unaids.org/en

2. McMichael AJ, Koff WC. Vaccines that stimulate $T$ cell immunity to HIV-1: the next step. Nat Immunol. (2014) 15:319-22. doi: 10.1038/ ni. 2844

3. Sok D, Burton DR. Recent progress in broadly neutralizing antibodies to HIV. Nat Immunol. (2018) 19:1179-88. doi: 10.1038/s41590-0180235-7

4. Julg B, Barouch DH. Neutralizing antibodies for HIV-1 prevention. Curr Opin HIV AIDS. (2019) 14:318-24. doi: 10.1097/COH.0000000000000556

5. Altfeld M, Kalife ET, Qi Y, Streeck H, Lichterfeld M, Johnston MN, et al. HLA alleles associated with delayed progression to aids contribute strongly to the initial CD8(+) T cell response against HIV-1. PLoS Med. (2006) 3:e403. doi: 10.1371/journal.pmed.0030403
Kenyatta National Hospital Ethical Review Committee of the University of Nairobi, the Rwanda National Ethics Committee, the Uganda Virus Research Institute Science and Ethics Committee (Currently the UVRI Research Ethics Committee) and the Uganda National Council of Science and Technology, the University of Cape Town Health Science Research and Ethics Committee, the Bio-Medical Research Ethics Committee at the University of KwaZulu Natal, the University of Zambia Research Ethics Committee, and the Emory University Institutional Review Board. Written informed consent was obtained for all participants. The patients/participants provided their written informed consent to participate in this study.

\section{AUTHOR CONTRIBUTIONS}

EMc and $\mathrm{JH}$ wrote the manuscript, provided conceptual input, and data analysis. AF-G, RR, DM, DCM, LY, and MN provided technical expertise and contributed to manuscript. JD, HC, and CS performed ELISPOT assays. DD, SB, AK, $\mathrm{GU}, \mathrm{GM}$, and $\mathrm{EMu}$ were integral to providing the input viral sequence data. EH and JG provided key supervision and support. All authors contributed to the article and approved the submitted version.

\section{ACKNOWLEDGMENTS}

This work was funded in part by IAVI and made possible by the support of the United States Agency for International Development (USAID) and other donors. The full list of IAVI donors is available at http://www.iavi.org. The contents of this manuscript are the responsibility of IAVI and do not necessarily reflect the views of USAID or the US Government. This paper was accepted for pre-print with BioRxiv (39).

\section{SUPPLEMENTARY MATERIAL}

The Supplementary Material for this article can be found online at: https://www.frontiersin.org/articles/10.3389/fimmu. 2021.609884/full\#supplementary-material
6. Ogishi M, and Yotsuyanagi H. Quantitative prediction of the landscape of $\mathrm{T}$ cell epitope immunogenicity in sequence space. Front Immunol. (2019) 10:827. doi: 10.3389/fimmu.2019.00827

7. Gaiha GD, Rossin EJ, Urbach J, Landeros C, Collins DR, Nwonu C, et al. Structural topology defines protective CD8 $+\mathrm{T}$ cell epitopes in the HIV proteome. Science. (2019) 364:480-4. doi: 10.1126/science.aav5095

8. Ondondo B, Murakoshi H, Clutton G, Abdul-Jawad S, Wee EGT, Gatanaga $\mathrm{H}$, et al. Novel conserved-region t-cell mosaic vaccine with high global HIV1 coverage is recognized by protective responses in untreated infection. Mol Ther. (2016) 24:832-42. doi: 10.1038/mt.2016.3

9. Baden LR, Walsh SR, Seaman MS, Cohen YZ, Johnson JA, Licona JH, et al. First-in-human randomized, controlled trial of mosaic HIV-1 immunogens delivered via a modified vaccinia ankara vector. J Infect Dis. (2018) 218:63344. doi: 10.1093/infdis/jiy212

10. Guardo AC, Joe PT, Miralles L, Bargalló M. E., Mothe B, Krasniqi A, et al. Preclinical evaluation of an mRNA HIV vaccine combining rationally selected 
antigenic sequences and adjuvant signals (HTI-TriMix). AIDS. (2016) 31:32133. doi: 10.1097/QAD.0000000000001276

11. Soria-Guerra RE, Nieto-Gomez R, Govea-Alonso DO, RosalesMendoza S. An overview of bioinformatics tools for epitope prediction: Implications on vaccine development. J Biomed Inform. (2015) 53:405-14. doi: 10.1016/j.jbi.2014.11.003

12. Kearney M, Maldarelli F, Shao W, Margolick JB, Daar ES, Mellors JW, et al. Human immunodeficiency virus type 1 population genetics and adaptation in newly infected individuals. J Virol. (2009) 83:271527. doi: 10.1128/JVI.01960-08

13. Li G, Piampongsant S, Faria NR, Voet A, Pineda-Peña AC, Khouri R, et al. An integrated map of HIV genome-wide variation from a population perspective. Retrovirology. (2015) 12:18. doi: 10.1186/s12977-015-0148-6

14. Taylor BS, Sobieszczyk ME, McCutchan FE, Hammer SM. The challenge of HIV-1 subtype diversity. $N$ Engl J Med. (2008) 358:1590-602. doi: 10.1056/NEJMra0706737

15. Maldarelli F, Kearney M, Palmer S, Stephens R, Mican J, Polis MA, et al. HIV populations are large and accumulate high genetic diversity in a nonlinear fashion. J Virol. (2013) 87:10313-23. doi: 10.1128/JVI.01225-12

16. Amornkul PN, Karita E, Kamali A, Rida WN, Sanders EJ, Lakhi S, et al. Disease progression by infecting HIV-1 subtype in a seroconverter cohort in sub-Saharan Africa. AIDS. (2013) 27:2775-86. doi: 10.1097/QAD.0000000000000012

17. Nielsen M, Lundegaard C, Blicher T, Lamberth K, Harndahl M, Justesen S, et al. NetMHCpan, a method for quantitative predictions of peptide binding to any HLA-A and -B locus protein of known sequence. PLoS ONE. (2007) 2:e796. doi: 10.1371/journal.pone.0000796

18. Nielsen M, Andreatta M. NetMHCpan-3.0; improved prediction of binding to MHC class I molecules integrating information from multiple receptor and peptide length datasets. Genome Med. (2016) 8:33. doi: 10.1186/s13073-016-0288-x

19. Joseph SB, Swanstrom R, Kashuba ADM, Cohen MS. Bottlenecks in HIV-1 transmission: insights from the study of founder viruses. Nat Rev Microbiol. (2015) 13:414-25. doi: 10.1038/nrmicro3471

20. Hare J, Fiore-Gartland A, Mcgowan E, Rosenthal R, Hunter E, Gilmour J, et al. Selective HLA restriction permits the evaluation interpretation of immunogenic breadth at comparable levels to autologous HLA. (2020). doi: 10.20944/preprints202008.0467.v1. [Epub ahead of print].

21. Simek MD, Rida W, Priddy FH, Pung P, Carrow E, Laufer DS, et al. Human immunodeficiency virus type 1 elite neutralizers: individuals with broad and potent neutralizing activity identified by using a high-throughput neutralization assay together with an analytical selection algorithm. J Virol. (2009) 83:7337-48. doi: 10.1128/JVI.00110-09

22. Baalwa J, Wang S, Parrish NF, Decker JM, Keele BF, Learn GH, et al. Molecular identification, cloning and characterization of transmitted/founder HIV-1 subtype A, D and A/D infectious molecular clones. Virology. (2013) 436:33-48. doi: 10.1016/j.virol.2012.10.009

23. Claiborne DT, Prince JL, Scully E, Macharia G, Micci L, Lawson B, et al. Replicative fitness of transmitted HIV-1 drives acute immune activation, proviral load in memory CD4 $+\mathrm{T}$ cells, and disease progression. Proc Natl Acad Sci USA. (2015) 112:E1480-9. doi: 10.1073/pnas.1421607112

24. Jurtz V, Paul S, Andreatta M, Marcatili P, Peters B, Nielsen M. NetMHCpan4.0: improved peptide-MHC class I interaction predictions integrating eluted ligand and peptide binding affinity data. J Immunol. (2017) 199:33608. doi: 10.4049/jimmunol.1700893

25. Reynisson B, Alvarez B, Paul S, Peters B, Nielsen M. NetMHCpan-4.1 and NetMHCIIpan-4.0: improved predictions of MHC antigen presentation by concurrent motif deconvolution and integration of MS MHC eluted ligand data. Nucleic Acids Res. (2020) 48:W449-54. doi: 10.1093/nar/gkaa379

26. Mónaco DC, Dilernia DA, Fiore-Gartland A, Yu T, Prince JL, Dennis $\mathrm{KK}$, et al. Balance between transmitted HLA preadapted and nonassociated polymorphisms is a major determinant of HIV-1 disease progression. J Exp Med. (2016) 213:2049-63. doi: 10.1084/jem.20151984
27. Carlson JM, Schaefer M, Monaco DC, Batorsky R, Claiborne DT, Prince J, et al. HIV transmission. Selection bias at the heterosexual HIV-1 transmission bottleneck. Science. (2014) 345:1254031. doi: 10.1126/science.1254031

28. Michelo CM, Dalel JA, Hayes P, Fernandez N, Fiore-Gartland A, Kilembe W, et al. Comprehensive epitope mapping using polyclonally expanded human CD8 T cells and a two-step ELISpot assay for testing large peptide libraries. $J$ Immunol Methods. (2021) 112970. doi: 10.1016/j.jim.2021.112970

29. Marsh SGE, WHO Nomenclature Committee for Factors of the HLA System. Nomenclature for factors of the HLA system, update April 2017. HLA. (2017) 90:188-92. doi: 10.1111/tan.13090

30. Goepfert PA, Lumm W, Farmer P, Matthews P, Prendergast A, Carlson JM, et al. Transmission of HIV-1 Gag immune escape mutations is associated with reduced viral load in linked recipients. J Exp Med. (2008) 205:100917. doi: $10.1084 /$ jem. 20072457

31. Fiore-Gartland A, Manso BA, Friedrich DP, Gabriel EE, Finak G, Moodie $Z$, et al. Pooled-peptide epitope mapping strategies are efficient and highly sensitive: an evaluation of methods for identifying human $\mathrm{T}$ cell epitope specificities in large-scale HIV vaccine efficacy trials. PLOS ONE. (2016) 11:e0147812. doi: 10.1371/journal.pone.0147812

32. Sidney J, Peters B, Frahm N, Brander C, Sette A. HLA class I supertypes: a revised and updated classification. BMC Immunol. (2008) 9:1. doi: 10.1186/1471-2172-9-1

33. Ngumbela KC, Ryan KP, Sivamurthy R, Brockman MA, Gandhi RT, Bhardwaj $\mathrm{N}$, et al. Quantitative effect of suboptimal codon usage on translational efficiency of mRNA encoding HIV-1 gag in intact T cells. PLoS One. (2008) 3:e2356. doi: 10.1371/journal.pone.0002356

34. Naarding MA, Fernandez N, Kappes JC, Hayes P, Ahmed T, Icyuz M, et al. Development of a luciferase based viral inhibition assay to evaluate vaccine induced CD8 T-cell responses. J Immunol Methods. (2014) 409:16173. doi: 10.1016/j.jim.2013.11.021

35. Spentzou A, Bergin P, Gill D, Cheeseman H, Ashraf A, Kaltsidis H, et al. Viral Inhibition assay: a CD8 T cell neutralization assay for use in clinical trials of HIV-1 vaccine candidates. J Infect Dis. (2010) 201:720-9. doi: 10.1086/650492

36. Kunwar P, Hawkins N, Dinges WL, Liu Y, Gabriel EE, Swan DA, et al. Superior control of HIV-1 replication by CD8 $+\mathrm{T}$ cells targeting conserved epitopes: implications for HIV vaccine design. PLoS ONE. (2013) 8:e64405. doi: 10.1371/journal.pone.0064405

37. Mothe B, Llano A, Ibarrondo J, Zamarreño J, Schiaulini M, Miranda $\mathrm{C}$, et al. CTL responses of high functional avidity and broad variant cross-reactivity are associated with HIV control. PLoS ONE. (2012) 7:e29717. doi: 10.1371/journal.pone.0029717

38. Sunshine J, Kim M, Carlson JM, Heckerman D, Czartoski J, Migueles SA, et al. Increased sequence coverage through combined targeting of variant and conserved epitopes correlates with control of HIV replication. J Virol. (2014) 88:1354-65. doi: 10.1128/JVI.02361-13

39. McGowan E, Rosenthal R, Fiore-Gartland A, Macharia G, Balinda S, Kapaata A, et al. Utilizing computational machine learning tools to understand immunogenic breadth in the context of a CD8 T-cell mediated 2 HIV response 3. bioRxiv. (2020). doi: 10.1101/2020.08.15.250589

Conflict of Interest: The authors declare that the research was conducted in the absence of any commercial or financial relationships that could be construed as a potential conflict of interest.

Copyright (c) 2021 McGowan, Rosenthal, Fiore-Gartland, Macharia, Balinda, Kapaata, Umviligihozo, Muok, Dalel, Streatfield, Coutinho, Dilernia, Monaco, Morrison, Yue, Hunter, Nielsen, Gilmour and Hare. This is an open-access article distributed under the terms of the Creative Commons Attribution License (CC BY). The use, distribution or reproduction in other forums is permitted, provided the original author(s) and the copyright owner(s) are credited and that the original publication in this journal is cited, in accordance with accepted academic practice. No use, distribution or reproduction is permitted which does not comply with these terms. 